\title{
Contaminación de los Parques Públicos de la Localidad de Suba, Bogotá con Nemátodos Zoonóticos
}

\author{
Zoonotic nematode contamination in recreational areas \\ of Suba, Bogotá
}

Luis J Polo-Terán ${ }^{1}$, Jesús A. Cortés-Vecino² ${ }^{2}$ Luis C. Villamil-Jiménez ${ }^{3}$ y

Edgar Prieto ${ }^{4}$

\begin{abstract}
1 Instituto Nacional de Salud, Colombia. luchopoloeb@yahoo.es
2 Departamento ciencias de la salud animal, Facultad de Medicina Veterinaria y Zootecnia.

Universidad Nacional de Colombia, Bogotá, Colombia. jacortesv@unal.edu.co

3 Universidad de la Salle. Colombia. lvillamil@jupiter.lasalle.edu.co

4 Departamento de Salud Pública, Facultad de Medicina. Universidad Nacional de Colombia,

Bogotá, Colombia. eprietos@unal.edu.co
\end{abstract}

Recibido 30 Enero 2007/Enviado para Modificación 30 Septiembre 2007/Aceptado 29 Octubre 2007

\section{RESUMEN}

Objetivo Establecer la presencia en los suelos de parques públicos de la localidad de Suba en la ciudad de Bogotá, de nemátodos gastrointestinales zoonóticos durante el periodo comprendido entre Julio de 2005 y Junio de 2006.

Metodología A través de una sedimentación inicial en suelo y posterior flotación con solución de Sheather, fue posible determinar la presencia de huevos, larvas y ooquistes de varios parásitos gastrointestinales, algunos de ellos responsables de importantes enfermedades zoonóticas.

Resultados Se identificados parásitos en 376 muestras que fueron descritos como huevos de Ancylostoma spp 10,7 \%, larvas de Ancylostoma spp 0,6 \%, huevos de Toxocara spp 5,4 \%, huevos de Strongyloides spp 3,3 \%, huevos de Dipylidium spp $0,1 \%$ y ooquistes de Sarcocystis spp 0,1\%. Otros parásitos identificados fueron huevos de Toxascaris spp 0,9\%, huevos de Spirocerca spp 0,3\%, ooquistes de Isospora spp 0,3\%. No fue posible clasificar algunas larvas y huevos en 522 muestras; no hubo evidencia de parásitos en 702 muestras $45 \%$. El 94,2 \% (n=49) de los parques estaban contaminados.

Conclusiones Lo anterior, indica que dichos parques constituyen un factor de riesgo para la adquisición de enfermedades parasitarias en los animales y los seres humanos. Debido a la relevancia de este problema de Salud Pública, es necesario instaurar políticas gubernamentales, involucrando la comunidad, educando las familias para mejorar sus prácticas sanitarias en lugares recreacionales con relación a la tenencia adecuada de los animales domésticos (mascotas).

Palabras Clave: Parásitos, zoonosis, animales domésticos (fuente: DeCS, BIREME). 


\section{ABSTRACT}

Objective Establishing the presence of zoonotic gastrointestinal nematodosin soil samples in recreational areas in Suba (one of the largest localities of Bogotá, Colombia) between July 2005 and June 2006.

Methodology The presence of several gastrointestinal parasites' eggs, larvae and oocysts were determined by initial soil sedimentation and later flotation with Sheather solution; some of them were responsible for important zoonotic diseases affecting the human population.

Results Parasites were identified in 376 samples: Ancylostoma larvae (10,7 \%), Ancylostoma eggs (0,6 \%), Toxocara eggs (5,8 \%), Strongyloides eggs (3,3\%), Dipylidium eggs $(0,06 \%)$ and Sarcocystis oocysts (0,06\%). Other parasites identified were Toxascaris eggs (0,9\%), Spirocerca eggs $(0,25 \%)$ and Isospora oocysts $(0,25$ $\%)$. Some larvae and eggs present in 522 samples could not be classified and there was no evidence of parasites in 702 samples (45\%). 94,2 \%, ( $n=49)$ of the recreational area was thus polluted.

Conclusions The high percentage of recreational areas so polluted indicated that these places would constitute a risk factor for the transmission of parasitic diseases to animals and human beings. Due to this sanitary problem's relevance in public health programmes, government policy must be determined involving communities so that people are educated to improve their sanitary practices in recreational places regarding caring for their pets.

Key Words: Parasite, recreational area, pets(fuente: DeCS,BIREME).

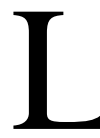

a mayoría de los brotes de enfermedades infecciosas ocurridos durante los últimos 10 años han incluido los agentes infecciosos zoonóticos $(1,2)$. rición de las infecciones humanas, lo cual no puede subestimarse dentro de su impacto en la salud de la comunidad y de allí la importancia de las mascotas como papel esencial en esta transmisión $(3,4)$. Las zoonosis parasitarias, tienen poca importancia dentro del contexto de la Salud Pública, ya que no dan lugar a emergencias epidemiológicas notables, y no están sujetas a notificación obligatoria en la mayoría de los países; por lo tanto, no se consideran problemas de salud pública. Estas zoonosis persisten y suponen una amenaza donde las condiciones de pobreza agravadas por el fenómeno de las migraciones, favorecen la transmisión y el arraigo de focos endémicos (5).

La prevalencia de muchos parásitos intestinales se ha mantenido constante en caninos callejeros (6), así, como la liberación de un gran número de estados larvarios parasitarios transmisibles en ambientes propicios, representando un factor de riesgo para la transmisión a los humanos (7-13). El agua, el suelo y 
REVISTADE SALUD PÚBLICA· Volumen 9 (4), Diciembre 2007

los alimentos llegan a tener una significancia particular en esta ruta de transmisión medio ambiental (14).

Las personas inmunocomprometidas, los niños, los adultos mayores, así como los propietarios de mascotas deben tener conocimiento sobre el riesgo potencial de adquirir infecciones parasitarias a partir de sus mascotas y de los sitios frecuentados por los animales (15).

La localidad de Suba en la ciudad de Bogotá, está catalogada como una zona de alto riesgo de presentación de enfermedades zoonóticas parasitarias, principalmente Larva Migrans Cutánea y Larva Migrans Ocular y Visceral y además constituye una zona de alto riesgo de presentación de enfermedades zoonóticas parasitarias según parámetros de población canina callejera, relación perro-hombre, prevalencia total de parásitos $(6,16)$.

En esta investigación, se propone medir el grado de contaminación de los sitios de esparcimiento público con las excretas de las mascotas, que frecuentan estos sitios. El estudio de la contaminación parasitaria del suelo está considerado como un indicador directo del riesgo de infección al que están expuestos los residentes de una región $(17,18)$.

\section{MATERIALES Y MÉTODOS}

Área de estudio

La localidad de Suba está ubicada en el sector norocccidental del distrito capital de Bogotá (Colombia). Su clima es frío subhúmedo, con temperatura promedio de $12,6{ }^{\circ} \mathrm{C}$ precipitación media anual de 900 a $1000 \mathrm{~mm}$ y una humedad relativa máxima de 77,6 \% (19).

Colección de muestras de suelo Las muestras se colectaron en bolsas plásticas durante el periodo comprendido entre Julio de 2005 y Junio de 2006. La unidad de estudio fueron las muestras de suelo de todos los parques de la localidad de Suba en la ciudad de Bogotá y la unidad de selección: parques (conglomerados).

Para el cálculo del tamaño de muestra, se plantea la estimación de una proporción, se hicieron los siguientes considerandos: prevalencia a priori en suelo ( $\mathrm{p}=20 \%$ ) tomado de un estudio previo ${ }^{1}$, aceptando para el estudio un

${ }^{1}$ Mosquera M [y otros] [Determinación del grado de contaminación de los suelos de 10 parques vecinales de la localidad de Suba con huevos de Toxocara]. Tesis de Grado [2004]. Se localiza en: Universidad Colegio Mayor de Cundinamarca Grupo 9. Cod Biblioteca: B 147. 
error absoluto a priori de $2 \%$ y un nivel de confianza del 95\% ( $\mathrm{t}=1.96)$. El tamaño de la muestra final fue de 1560 unidades de estudio. Para la toma de estas unidades de estudio se definieron unidades de muestreo (parques de la localidad), tomando en cada parque un número de 30 unidades de estudio, debiendo tener para seleccionar 52 parques $(1560 / 30=52)$. En la localidad hay 348 distribuidos en los diferentes estratos socieconómicos. Se realizó una selección aleatoria con asignación proporcional de estratos para los 52 parques.

La unidad de estudio se definió como el volumen de suelo resultante de recoger con una pala una porción de: $10 \mathrm{~cm}$ de largo, por $10 \mathrm{~cm}$ de ancho y por $3 \mathrm{~cm}$ de profundidad. En cada parque se tomó una muestra cada $100 \mathrm{~m}^{2}$, para poder tener un barrido completo del parque para el estudio de la posible contaminación (11). Las muestras se colectaron en bolsas plásticas, y se guardaron a 4 grados centígrados para conservar sus características hasta su procesamiento dentro de las 72 horas siguientes.

Análisis de las muestras de suelos

Las muestras se procesaron por la Técnica de Sloss que emplea el reactivo de Sheather (técnica de sedimentación y de flotación para la determinación de huevos y estados larvarios de helmintos) según el protocolo del Laboratorio de Parasitología de la Facultad de Medicina Veterinaria y de Zootecnia (20). Se consideraron positivas las muestras que presentaron al menos un huevo de nemátodos gastrointestinales, quistes o larvas identificados mediante claves parasitológicas.

Análisis de parámetros cualitativos

A la par de la recolección de muestras se hicieron observaciones en relación con los parques, así: presencia de juegos infantiles, estado de conservación, señalización, encerramiento, contaminación con materia fecal y presencia de mascotas.

Análisis de información

Se realizó una base de datos con Epi-Info 2000 versión 3.3.2. De la información recolectada se determinó la proporción muestral para cada parque y para el total. La estimación de la prevalencia se hizo con un nivel de confianza del 95\%.

\section{RESULTADOS}

De las 1560 muestras analizadas 24,1 \% (n= 376) (IC: 21,9-26,2\%) presentaban parásitos gastrointestinales. Aunque el diseño del estudio (prevalencia a priori) 
REVISTADE SALUD PÚBLICA · Volumen 9(4), Diciembre 2007

era en relación con positivismo en la muestra para parásitos gastrointestinales, en la Tabla 1 se indica la proporción de parásitos identificados.

Tabla 1. Resultados generales

\begin{tabular}{lcc}
\hline Parásito & No. Muestras (+) & Porcentaje (\%) \\
\hline Ancylostómidos & 176 & 11,3 \\
Toxocara spp & 84 & 5,4 \\
Toxascaris spp & 14 & 0,9 \\
Strongyloides spp & 52 & 3,3 \\
Sarcocystis spp & 1 & 0,1 \\
Isospora spp & 4 & 0,3 \\
Dipylidium spp & 1 & 0,1 \\
Spirocerca spp & 4 & 0,3 \\
\hline
\end{tabular}

Del total de las muestras positivas para Ancylostoma spp, $95 \%$ correspondieron a huevos y $5 \%$ restante a larvas (cutícula envainada).

La positividad reportada en este estudio para Toxocara spp en las muestras de suelo, fue de 5,4 \% del cual el total correspondían a huevos con presencia de la larva bien formada en su interior.

De los parques públicos investigados en este trabajo 94,2 \% ( $\mathrm{n}=49)$ contenían formas infectivas de parásitos intestinales. La mayoría presentaban un aspecto entre regular y bueno según la calificación propuesta por varios autores $(21,22)$. La característica común en todos los parques estudiados era la presencia de juegos infantiles.

La contaminación fue evidente en los parques tuvieran o no la señalización de prevención sobre el uso del parque. Además, se observó gran cantidad de materia fecal canina y humana en sus zonas verdes.

\section{DISCUSIÓN}

La baja prevalencia de Ancylostoma spp en este estudio, comparada con otros reportes, puede deberse a la humedad relativa reinante en la localidad que no permiten un desarrollo óptimo de los huevos y larvas (23-26). Además, la presencia de larvas en sus diferentes estados, explican las malas condiciones higiénico-sanitarias y la contaminación presentes en dichos suelos, producto de la proliferación de perros callejeros en esta localidad demostrado en estudios anteriores (16).

Las prevalencias encontradas de Toxocara spp entre las muestras de materia fecal de caninos callejeros de la localidad de Suba, con un 5,8 \%, y las muestras de suelo de sus parques públicos, con un 5,4\%, están de acuerdo con 
la resistencia de los huevos de parásito a los factores medio ambientales (2326). Los huevos eliminados en las heces de sus hospederos definitivos, bajo condiciones adecuadas de temperatura y humedad, no propias de la zona de nuestro estudio, llegaron a conservarse como huevos larvados o embrionados infecciosos. La positividad por Toxocara spp, resultó ser más baja que la reportada por Fonrouge (8), Snow (27), Uga (28), Woodruff (29) y O'Larcain (30). Los resultados fueron muy similares a los reportados por Wiwanitkit (31) y Sommerfelt $(11,12)$.

Los parques mejor conservados son los más contaminados, ya que presentan la mayor proporción de áreas con vegetación, lo que proporciona las condiciones óptimas de humedad, temperatura y sombra que favorecen la supervivencia de los huevos de Toxocara spp, y larvas de ancylostómidos; mientras que los parques en mal estado de conservación, los huevos y larvas están expuestos a la desecación y a la acción directa de los rayos solares que los destruirían en corto tiempo; esto corrobora los resultados reportados por otros autores trabajando en similares condiciones medioambientales $(21,22)$.

Los resultados arrojados en este estudio indican que los parques públicos de la localidad de Suba, constituyen sitios de riesgo de infección con huevos de Toxocara spp (Larva Migrans Visceral u Ocular) y larvas infectantes de Ancylostoma spp (Larva Migrans Cutánea), para las personas que concurren a ellos (15). Se hace necesario llamar la atención a los organismos encargados de la administración de estos sitios para que tomen las medidas de educación sobre el manejo de las mascotas en espacios recreativos y de control de las mismas

Agradecimientos. A los integrantes de la oficina del Medio Ambiente del Hospital de Suba, a los ingenieros Zoraida Acosta y Pablo Parra del Instituto Distrital de Recreación y Deportes. Al laboratorio de Parasitología de la Facultad de Medicina Veterinaria y Zootecnia de la Universidad Nacional de Colombia.

\section{REFERENCIAS}

1. Instituto Colombiano Agropecuario/Organización Panamericana de la Salud. Salud Pública Veterinaria, protección sanitaria, y desarrollo agropecuario. Simposio Internacional. Memorias; Bogotá, Colombia; 11 de junio 2002.p 83-87.

2. Institute of Medicine (IOM): The Emergent of Zoonotic Diseases - Understanding the impact Animal and Human Health. Washington, DC National Academy Press; 2002. 
REVISTADE SALUD PÚBLICA· Volumen 9 (4), Diciembre 2007

3. Organización Panamericana de la Salud. Zoonosis y enfermedades transmisibles comunes al hombre y a los animales: parasitosis. Tercera edición. Washington, DC. OPS; Publicación científica técnica No 580; 2003. p844-849.

4. Ambroise-Thomas P. Emerging parasite zoonoses: the role of host-parasite relationship. International Journal of Parasitology. 2000; 30: 1361-1367.

5. Organización Panamericana de la Salud/Organización Mundial de la Salud. 14 Reunión Interamericana a Nivel Ministerial en Salud y Agricultura. RIMSA. Las enfermedades desatendidas en las poblaciones postergadas, con énfasis en las zoonosis. Ciudad de México; 18 abril 2005.

6. Cabrera PA, Ordóñez OE. Prevalencia de Parásitos Gastrointestinales Zoonóticos (Helmintos y Protozoarios) en caninos del Centro de Zoonosis de Bogotá. Investigaciones en Seguridad Social y Salud. Secretaria Salud. Alcaldía Mayor de Bogotá. 2004; (6): 71-93.

7. Córdoba A, Ciarmela M, Pezzari B, Gamboa M, De Luca M, Minvelle M, etal. Presencia de parásitos intestinales en paseos públicos urbanos en la Plata Argentina. Parasitología Latinoamericana. 2002; 57: 25-29.

8. Fonrouge R. Contaminación de suelos con huevos de Toxocara sp. en plazas y parques públicos de la ciudad de La Plata. Buenos Aires, Argentina. Boletín Chileno de Parasitología. 2000; 55: 83-85.

9. Sanchez P, Ñancufil A, Oyarzo C, Torrecillas C, Raso S, Mellado I, et al. An ecoepidemiological study of contamination of soil with infective forms of intestinal parasites. European Journal of Epidemiology. 2004; 19: 481-489.

10. Sommerfelt I, DeGregorio O, Álvarez A, Gallo G, Franco AJ. Viabilidad de huevos de Toxocara canis. Revista de Medicina Veterinaria. 1995; 77 (4): 302-304.

11. Sommerfelt I, DeGregorio O, Barrera MY, Gallo G. Presencia de huevos de Toxocara spp. en paseos públicos de la ciudad de Buenos Aires, Argentina, 1989-90. Revista de Medicina Veterinaria, 1992; 73(2): 70-74.

12. Sommerfelt I, DeGregorio O, Barrera M, Gallo G Betti A. Contaminación Ambiental Urbana con Huevos de Endoparásitos de Origen Animal. Veterinaria Argentina, 1994; 11(107):457-461.

13. Taranto N, Passamonte L, Marinconz R, Marzi M. Zoonotic Parasitosis Transmitted By Dog InThe Chaco, Salteño, Argentina. Medicina (BUENOS AIRES). 2000; 60 (2): 217-220.

14. Slifko T, Smith H, Rose J. Emerging parasite zoonoses associated with water and food. International Journal of Parasitology, 2000; 30:1379-1393.

15. Robertson ID. The role of companion animals in the emergence of parasitic zoonoses. International Journal for Parasitology. 2000; 30:1369-1377.

16. Fundación Veterinaria para la Seguridad Social de la Mascota (FVSSM). Análisis de la Población canina en el Distrito Capital 2005. Informe final. Bogotá D.C.; Junio de 2005. 
17. Asaoulu S, Holland C, Jegede J. The prevalence and intensity of soil- transmited helminthiases in rural communities in Southern Nigeria. Annual Tropic Medicine Parasitology. 1992; 86:278-87.

18. Uga S, Nagnae W, Chongsuvivatwong V. Contamination of soil with parasite eggs and oocysts in Southern Thailand. Southeast Asian Journal of Medicine and Public Health. 1997; 28: 14-17.

19. Secretaría Distrital de Salud. Alcaldía Mayor de Santafe de Bogotá. Diagnósticos Locales con Participación Social. Localidad-Suba. 1998; 958(8069). p 15-17.

20. Laboratorio de Parasitología Veterinaria. Protocolos de Laboratorio. Facultad de Medicina Veterinaria y Zootecnia. Universidad Nacional de Colombia. Bogotá; 2005.

21. Chávez A, Casas E, Cajas J, Velarde J. Contaminación de parques públicos con huevos de Toxocara spp en los distritos de la provincia constitucional del Callao y del Cono Sur de Lima metropolitana. Revista de Investigaciones Veterinarias del Perú. 2000; 11(1):52-57.

22. La Rosa V, ChávezA, Casas E. Contaminación de Parques públicos del Cono Norte con huevos de Toxocara spp. Revista de Investigaciones Veterinarias del Perú; 1999.

23. Cordero Del Campillo M. Parasitología Veterinaria. Madrid. McGraw Hill. Interamericano; 1999.

24. Urquhart GM. Veterinary Parasitology. Second Edition. Blackwell Science Ltd. Scotland; 1996.

25. Laird R, Carballo D, Reyes E, García R, Prieto V. Toxocara spp en parques y zonas públicas de Ciudad de la Habana, 1995. Revista Cubana de Higiene y Epidemiología. Ciudad de la Habana. 2000; 38(2): 112-116.

26. O’Donnell CJ, Meyer KB, Jones J, Benton T, Kaneshiro E, Nichols J, et al. Survival of parasite eggs upon storage in sludge. Appl Environment Microbiology. 1984; 48 (3):618-625.

27. Snow K, Ball S, Bewick J. Prevalence of Toxocara species eggs in the soil of five east London parks. Veterinary Record. 1987; 120: 66-67.

28. Uga S, Kataoka N. Measures to control Toxocara eggs contamination in sandpits of public parks. American Journal Tropic Medicine and Hygiene. 1995; 52: 21-24.

29. Woodruff AW, Watson, Shikara L, AlAzzi N, Al Adhami S, Woodruff P.. Toxocara ova in soil in the Mosul District, Iraq, and their relevance to public to public health measures in the Middle East. Annual Tropic Medicine Parasitology. 1981; 75:5557.

30. O'Larcain P. Prevalence of Toxocara canis ova in public playgrounds in the Dublin area of Ireland. Journal Helminthology. 1994; 68: 237-241.

31. Wiwanitkit V, Waenlor W. The Frequency rate of Toxocara species Contamination in soil samples from public yards in a urban area "Payathai", Bangkok, Thailand. Revista do Instituto do Medicina Tropical de Sao Paulo. 2004; 46(2): 113-114. 